\title{
Aminotransferases Screening of Nitro-D-Arginine Using Molecular Modeling
}

\author{
Xiangfei Yang ${ }^{1}$, Shupeng Liu ${ }^{1+}$, Na Chen ${ }^{1}$, Zhenyi Chen ${ }^{1}$, Yanfei Xin ${ }^{2}$, Heng Zhang ${ }^{1}$, Taihao Li $^{3}$ \\ ${ }^{1}$ Key Laboratory of Specialty Fiber Optics and Optical Access Networks, School of Communication and \\ Information Engineering, Shanghai University, 333 Nanchen Road, Shanghai 200444, China \\ ${ }^{2}$ State Key Laboratory of Safety Evaluation for New Drugs, Zhejiang Academy of Medical Sciences, \\ Hangzhou, Zhejiang, 310013, China \\ ${ }^{3}$ Beijing Advanced Innovation Center for Imaging Technology, Capital Normal University, Beijing 100048, \\ China.
}

\begin{abstract}
This study is intended to screen the potential aminotransferases using molecular modeling technique. In detail, the sybyl-x software was employed to simulate the second step of catalysis process of nitro-D-arginine (D-NNA) by aminotransferases whose structural data were from Protein Data Bank (PDB). Results showed the most suitable candidate aminotransferase was human mitochondrial aspartate aminotransferase in the range of our research, which might aid to shortening the drug developing cycle and increasing the efficiency of lead compound discovery.
\end{abstract}

Keywords: molecular modeling, aminotransferase screen, nitro-D-arginine

\section{Introduction}

Molecular modeling is widely used in drug developing, material design and other fields [1]. As a powerful tool, molecular modeling provides the exploration guideline for the drug design in a simulation way, calculating the biomolecular interactions on the computer with saving time and money. There are many molecular modeling softwares were developed such as Affinity, Dock, DDT, AutoDock, DOCK, 3D-DOCK, ZDOCK, GRAMM, DOT, Modified, and FTDOCK [2, 3]. For example, DOCK software developed by Kuntz group, has the capacity of identification for the low-energy binding modes of a small molecule, or ligand, within the active site of a macromolecule, or receptor, whose structure is known $[4,5]$.

Studies reported that nitro-D-arginine could be converted into nitro-L-arginine as an inhibitor of nitric oxide synthase by chiral switch [6-9]. D- amino acid could be catalyzed into $\alpha$-keto acid by the D- amino acids oxidase, which mainly distributed in the kidney in mammals. It was speculated that $\alpha$-keto acids were converted to nitro-L-arginine by aminotransferase [7].

In this paper, the molecular docking software Sybyl was employed to simulate the molecular docking for the possible conversion of nitro-D-arginine to nitro-L-arginine, and screen the transaminase based on the scoring function.

\section{Methods}

The 3D data of transaminase structure were from Protein Data Bank (PDB, http://www.rcsb.org), which collects the 3D shapes of proteins, nucleic acids, and complex assemblies ${ }^{[1]}$. The transaminase was selected as the target protein molecule (Table 1).

Corresponding author. Tel.: + 86-21-66137229; fax: +86-21-66137229.

E-mail address: liusp@shu.edu.cn 
Table 1: The main transaminase selected

\begin{tabular}{ll}
\hline PDB ID & Candidate Aminotransferase \\
\hline 5AX8 & human mitochondrial aspartate aminotransferase \\
3IHJ & Human alanine aminotransferase 2 \\
3DYD & Human Tyrosine Aminotransferase \\
2BYJ & Ornithine aminotransferase mutant Y85I \\
3E77 & Human phosphorserine aminotransferase \\
3II0 & human Glutamate oxaloacetate transaminase 1 \\
4KYO & Alanine-glyoxylate aminotransferase variant K390A \\
\hline
\end{tabular}

The small ligand molecules were removed from the structure of the aminotransferase using software sybyl-x. For example, the PLP were deleted from the PDB data of 5AX8 (Fig. 1). Then the aminotransferase were analyzed to dock with $\alpha$-keto acid.

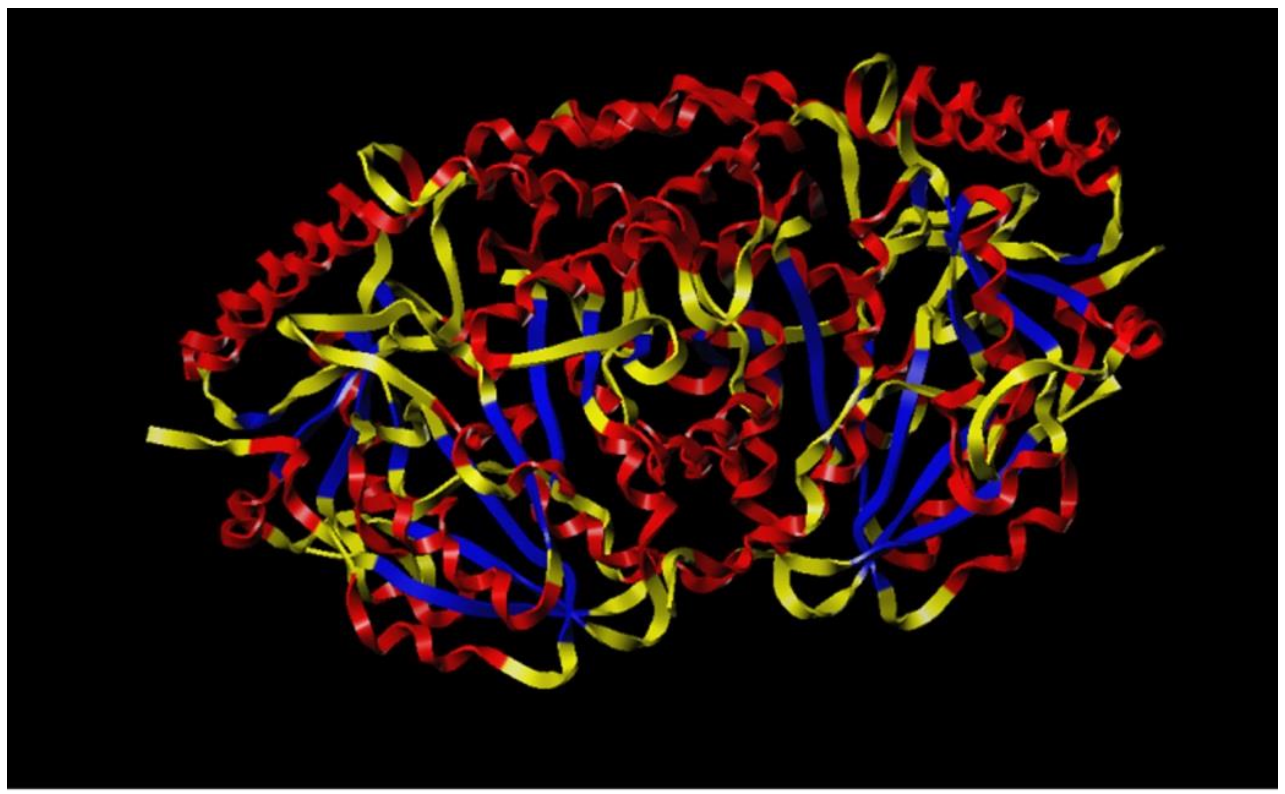

Fig. 1: human mitochondrial aspartate aminotransferase (5AX8)

$\alpha$-keto acid were prepared by chemical paint tools in Sybyl, and the hydrogen atom was added to the protein structures (Fig. 2). The rigid rood and rotational bond were saved for the flexible docking.

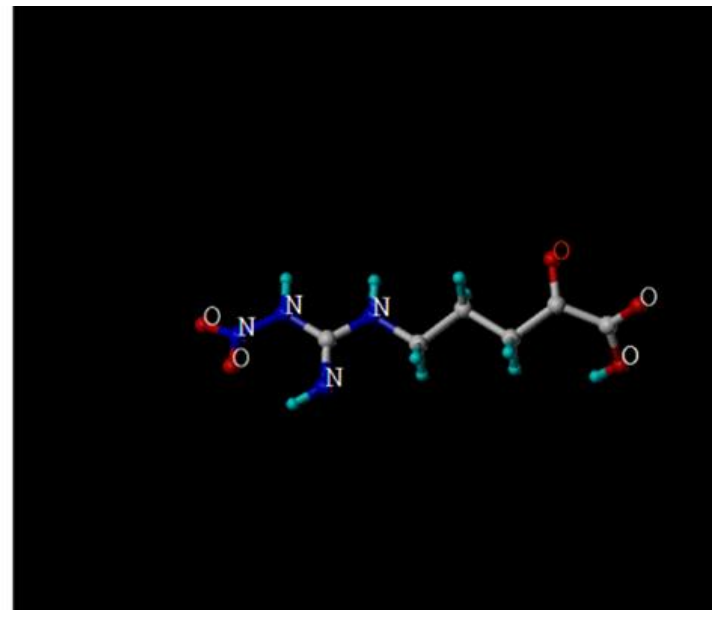

Fig. 2: the molecular model of $\alpha$-keto acid

In molecular docking, small molecule ligands corresponding to the receptor are needed to be preprocessed. Generally, the changed-adding and hydrogasification processing on the terminal should be performed beforehand, and then minimized the molecular structure energy using powell energy gradient algorithm, with the convergence limit of $0.5 \mathrm{cal} / \mathrm{mol}$. The Tripos force field and atom charge were iterated 
using Gasteiger-Huckel, and the other parameters were setup as the default values of Sybyl software for the mini-energy structure of molecular docking.

The ligand molecular were also processed by adding hydrogen and parameters setup as the proteins molecular processing and saved as the mol2 format.

\section{Results and Discussions}

The docking experiment for transaminase and nitro-D-arginine were carried using Sybyl software.

Table 2: The results of molecular docking

\begin{tabular}{|c|c|c|c|c|c|c|c|}
\hline Name & Total_Score & Crash & Polar & D_SCORE & $P M F \_S C O R E$ & CHEMSCORE & CSCORE \\
\hline NRG_001 & 8.6406 & -0.7921 & 8.1595 & -78.6878 & -87.9116 & -26.337 & 3 \\
\hline NRG_002 & 8.1465 & -0.9949 & 7.528 & -81.9974 & -99.2282 & -21.6335 & 3 \\
\hline NRG_003 & 7.9776 & -1.0021 & 6.9329 & -100.463 & -71.0198 & -22.376 & 3 \\
\hline NRG_004 & 7.9502 & -0.3757 & 6.547 & -88.6708 & -102.874 & -18.6506 & 2 \\
\hline NRG_005 & 7.7346 & -0.7818 & 6.8886 & -101.209 & -75.096 & -21.4337 & 3 \\
\hline NRG_006 & 7.2551 & -1.3402 & 7.3879 & -76.4041 & -89.7335 & -24.4206 & 2 \\
\hline NRG_007 & 7.2543 & -1.5509 & 7.2714 & -88.6045 & -72.4366 & -20.134 & 0 \\
\hline NRG_008 & 7.0521 & -1.1924 & 5.956 & -102.68 & -72.6075 & -19.7567 & 2 \\
\hline NRG_009 & 7.0299 & -0.5803 & 5.7346 & -80.4304 & -74.8378 & -17.8354 & 0 \\
\hline NRG_010 & 6.8278 & -0.6317 & 5.6184 & -80.7332 & -77.6624 & -17.4975 & 0 \\
\hline NRG_011 & 6.7106 & -1.3753 & 6.2685 & -80.8766 & -74.2781 & -19.0144 & 0 \\
\hline NRG_012 & 6.7097 & -1.6915 & 6.2622 & -95.3702 & -74.4473 & -15.3024 & 1 \\
\hline NRG_013 & 6.6759 & -1.8697 & 6.0955 & -109.848 & -68.8009 & -18.4744 & 2 \\
\hline NRG_014 & 6.631 & -1.2177 & 5.3637 & -94.19 & -68.5063 & -20.1126 & 2 \\
\hline NRG_015 & 6.6236 & -0.4761 & 5.4376 & -81.2247 & -99.6563 & -15.549 & 1 \\
\hline NRG_016 & 6.6195 & -1.1871 & 5.5621 & -104.382 & -69.4228 & -22.1755 & 3 \\
\hline NRG_017 & 6.5417 & -0.8144 & 7.846 & -73.6594 & -95.2494 & -24.1915 & 2 \\
\hline NRG_018 & 6.515 & -0.8882 & 6.2464 & -85.4758 & -96.7722 & -20.105 & 1 \\
\hline NRG_019 & 6.5119 & -1.2859 & 6.6463 & -74.5796 & -86.6566 & -22.6092 & 2 \\
\hline NRG_020 & 6.5003 & -0.7946 & 6.2276 & -81.8358 & -96.8135 & -19.6592 & 1 \\
\hline
\end{tabular}
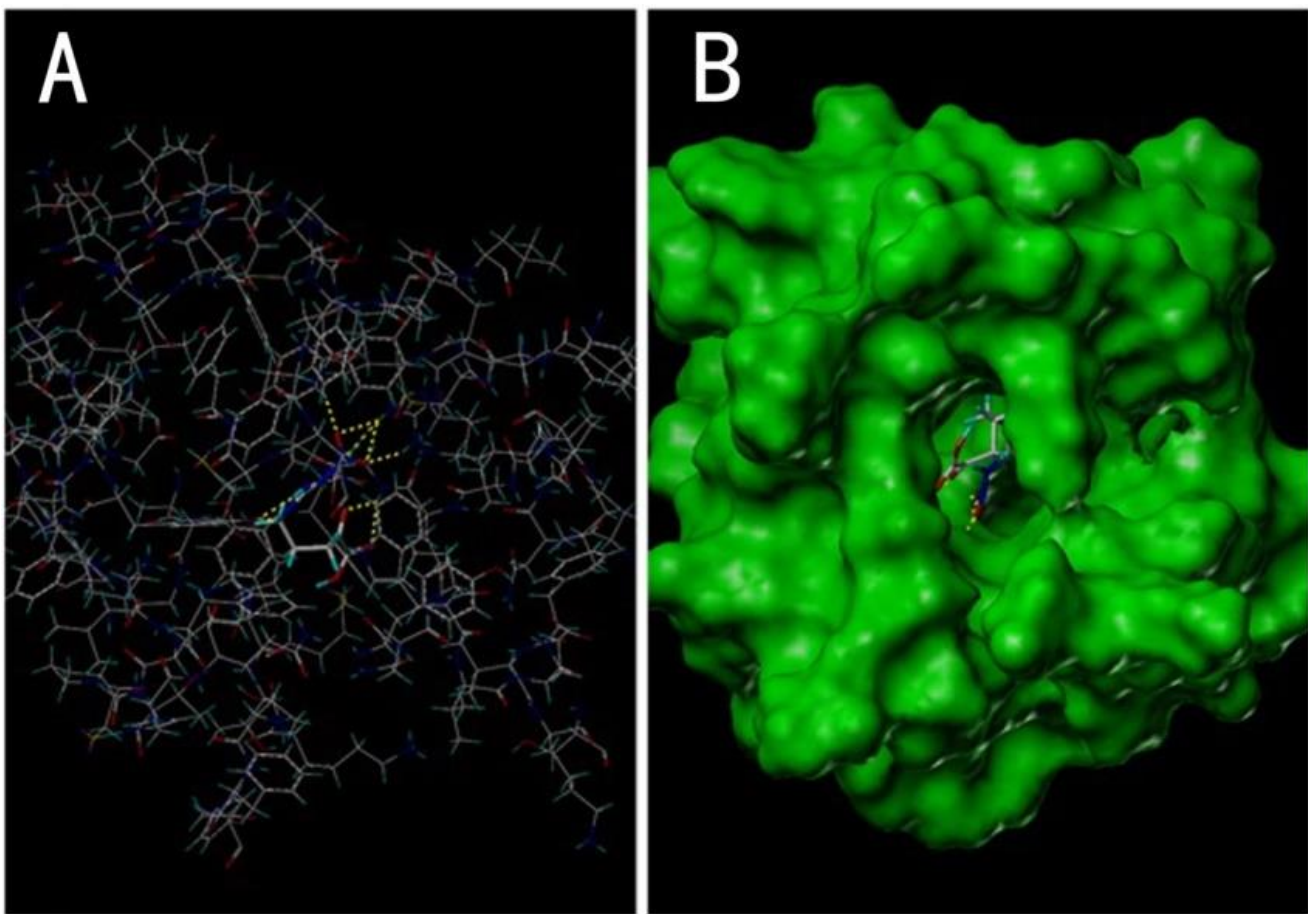

Fig. 3: The docking image of transaminase $\mathrm{K}$ and nitro-D-arginine. (A) Original structure of molecular docking model; (B) Structure model of rendering for the molecular 
Depended on the molecular modeling simulation and analysis (Table 2), in the second stage of the catalysis reaction of $\mathrm{N}^{\mathrm{G}}$-nitro-D-Arginine into enantiomers nitro-L-Arginine, the most suitable candidate aminotransferase was calculated out to be human mitochondrial aspartate aminotransferase (Fig. 3 ) in the range of our research.

\section{Acknowledgements}

This paper was funded by Natural Science Foundation of China (NSFC) (61422507, 61475095, 61520106014), and thanks for the support of the Key Laboratory of Specialty Fiber Optics and Optical Access Networks (SKLSFO2015-06). And this paper was supported by Beijing Advanced Innovation Center for Imaging Technology, and thanks for the software supporting of the Yonghua Ji group.

\section{References}

[1] H. M Berman. 'The Protein Data Bank: A Historical Perspective', in Acta Cryst (2008), pp. 88-95.

[2] E Yuriev, and P. A. Ramsland. 'Latest Developments in Molecular Docking: 2010-2011 in Review', Journal of Molecular Recognition Jmr, 26 (2013), 215-239.

[3] E Kellenberger, J Rodrigo, P Muller, et al. 'Comparative Evaluation of Eight Docking Tools for Docking and Virtual Screening Accuracy', Proteins-structure Function \& Bioinformatics, 57 (2004), 225-242.

[4] Helen M. Berman, Peter W. Rose, Shuchismita Dutta, et al. 'The Protein Data Bank: Overview and Tools for Drug Discovery', Nato Science for Peace \& Security, 38 (2015), 93-106.

[5] M Agostino, C Jene, T Boyle, et al. 'Molecular Docking of Carbohydrate Ligands to Antibodies: Structural Validation against Crystal Structures', Cheminform, 49 (2009), 2749-2760.

[6] Z. You, Y. Xin, Y. Liu, et al. 'Protective Effect of Salvia Miltiorrhizae Injection on N(G)-Nitro-D-Arginine Induced Nitric Oxide Deficient and Oxidative Damage in Rat Kidney', Experimental \& Toxicologic Pathology, 64 (2012), 453-458.

[7] X Yan-Fei, Z Xiang-Jun, L. Jie, et al. 'Inhibitory Effects of Benzoate on Chiral Inversion and Clearance of $\mathrm{N}(\mathrm{G})$-Nitro-Arginine in Conscious Rats', Drug Metabolism \& Disposition the Biological Fate of Chemicals, 35 (2007), 331-334.

[8] Yan Fei Xin, Li Xin, Bin Hao, et al. 'Indispensable but Insufficient Role of Renal D-Amino Acid Oxidase in Chiral Inversion of Ng-Nitro-D-Arginine', Chemistry \& Biodiversity, 7 (2010), 1413-1423.

[9] Y. X. Wang, N. Gong, Y. F. Xin, et al. 'Biological Implications of Oxidation and Unidirectional Chiral Inversion of D-Amino Acids', Current Drug Metabolism, 13 (2012), 321-331. 